\title{
Dø results on three-jet production, multijet cross-section ratios, and minimum bias angular correlations
}

\author{
Lee Sawyer ${ }^{* i+t}$ \\ Louisiana Tech University \\ E-mail: sawyer@latech.edu
}

\begin{abstract}
We report the measurement of the cross-section for three-jet production and the ratio of inclusive three-jet to two-jet cross-sections, as well as a study of angular correlations in minimum bias events, based on data taken with the $\mathrm{D} \emptyset$ experiment at the Fermilab Tevatron proton-antiproton collider.

The differential inclusive three-jet cross section as a function of the invariant three-jet mass $\left(M_{3 \mathrm{jet}}\right)$ is measured in $p \bar{p}$ collisions at $\sqrt{s}=1.96 \mathrm{TeV}$ using a data set corresponding to an integrated luminosity of $0.7 \mathrm{fb}^{-1}$. The measurement is performed in three rapidity regions $(|y|<0.8,|y|<$ 1.6 and $|y|<2.4)$ and in three regions of the third (ordered in $\left.p_{T}\right)$ jet transverse momenta $\left(p_{T 3}>\right.$ $40 \mathrm{GeV}, p_{T 3}>70 \mathrm{GeV}, p_{T 3}>100 \mathrm{GeV}$ ) for events with leading jet transverse momentum larger than $150 \mathrm{GeV}$ and well separated jets. NLO QCD calculations are found to be in a reasonable agreement with the measured cross sections.

Based on the same data set, we present the first measurement of ratios of multi-jet cross sections in $p \bar{p}$ collisions at $\sqrt{s}=1.96 \mathrm{TeV}$ at the Fermilab Tevatron Collider. The ratio of inclusive trijet and dijet cross sections, $R_{3 / 2}$, has been measured as a function of the transverse jet momenta. The data are compared to QCD model predictions in different approximations.

Finally, we present a new way to describe minimum bias events based on angular distributions in $\approx 5$ million minimum bias $p \bar{p}$ collisions collected between April 2002 and February 2006 with the $\mathrm{D} \emptyset$ detector. We demonstrate that the distribution of $\Delta \phi$ in the detector transverse plane between the leading track and all other tracks is a robust observable that can be used for tuning of multiple color interaction models. Pseudorapidity correlations of the $\Delta \phi$ distributions are also studied.
\end{abstract}

XVIII International Workshop on Deep-Inelastic Scattering and Related Subjects

April 19-23, 2010

Convitto della Calza, Firenze, Italy

*Speaker.

${ }^{\dagger}$ My thanks to the DØ QCD physics group conveners (Don Lincoln, Dmitry Bandurin, Sabine Lammers) and to Markus Wobisch, Zdenek Hubacek, Camille Belanger-Champagne and Claus Buszello for their help preparing this talk.

${ }^{\ddagger}$ Delivered via Skype for BlackBerry from the Hotel Atena De Mar, Barcelona, Spain. If you are going to be stranded due to eruptions of the Eyjafjallajökull volcano, Barcelona is not a bad place to be. 


\section{Introduction}

In hadron-hadron collisions, production rates of collimated sprays of hadrons, called jets, are sensitive to both the dynamics of the fundamental interaction and to the partonic structure of the initial-state hadrons. Thus measurements of jet production properties can be used to test the predictions of quantum chromodynamics (QCD) as well as to constrain the PDFs.

While jet production is amenable to tests using pertubative $\mathrm{QCD}$, there are many other processes at a hadron collider in which non-perturbative QCD predominates. It is also important to develop tests of the heuristic models that have been developed for non-perturbative processes, in order to compare them in detail to experimental results.

\section{Multijet Measurements}

In the jet analyzes presented, we measure the trijet cross-section as a function of the invariant mass $M_{3 \text { jet }}$ of the three-jet system, and study the ratio of the inclusive trijet and dijet cross sections, in $p \bar{p}$ collisions at a center-of-mass energy of $\sqrt{s}=1.96 \mathrm{TeV}$, collected with the $\mathrm{D} \emptyset$ detector and corresponding to an integrated luminosity of $0.7 \mathrm{fb}^{-1}$.A detailed description of the D0 detector can be found in Ref. [1]. Jets are defined by the Run II midpoint cone jet algorithm [2] with a cone radius of $R_{\text {cone }}=\sqrt{(\Delta y)^{2}+(\Delta \phi)^{2}}=0.7$ in rapidity $y$ and azimuthal angle $\phi$.

Jets in the inclusive $n$-jet event sample (for $n=2,3$ ) must have $p_{T}>p_{T \min },|y|<2.4$, for which the separation in the plane of rapidity and azimuthal angle, $R_{\mathrm{jj}}$, between all pairs of the $n$ leading $p_{T}$ jets is larger than twice the cone $\operatorname{radius}\left(R_{\mathrm{jj}}>2 \cdot R_{\text {cone }}\right)$.

We measure the differential inclusive three-jet cross section as a function of the invariant threejet mass for three hard, well separated jets in three regions of jet rapidities $(|y|<0.8,|y|<1.6$, $|y|<2.4$ for all three jets) and in three regions of the third jet transverse momentum $\left(p_{T 3}>40 \mathrm{GeV}\right.$, $p_{T 3}>70 \mathrm{GeV}$ and $p_{T 3}>100 \mathrm{GeV}$ ) with leading jet $p_{T}>150 \mathrm{GeV}$. The three-jet mass spectra are shown in Fig. 1.

The data are compared to the perturbative QCD NLO predictions calculated in NLOJET++[3] program with MSTW2008NLO[4] PDFs. The NLO prediction is corrected for hadronization and underlying event effects, with correction varied in the range from $-3 \%$ to $6 \%$, obtained from PYTHIA[5] simulations using tune QW[6]. The comparison of data to theory is shown in Fig. 2.

The ratio of inclusive jet cross sections, $R_{3 / 2}\left(p_{T \max }\right)=\left(d \sigma_{\geq 3 \text { jet }} / d p_{T \max }\right) /\left(d \sigma_{\geq 2-\text { jet }} / d p_{T \max }\right)$, is less sensitive to experimental and theoretical uncertainties than the individual cross sections, due to cancelations of correlated uncertainties. Here $R_{3 / 2}$ is measured as a function of the leading jet $p_{T}$ in an event, $p_{T \max }$, in the interval $\left(p_{T \min }+30 \mathrm{GeV}\right)<p_{T \max }<500 \mathrm{GeV}$, for $p_{T \text { min }}$ requirements of 50,70 , and $90 \mathrm{GeV}$.

The results are displayed in Fig. 3, where the inner error bars represent the statistical uncertainties while the total error bars represent the quadratic sums of statistical and systematic uncertainties. The data are compared to the predictions from different Monte Carlo event generators. The SHERPA v1.1.3 predictions [7], which include the tree-level matrix elements for 2-, 3-, and 4-jet production, are shown as solid lines in Figs. 3. These were obtained using default settings and MSTW2008LO PDFs and by matching the leading order matrix elements for 2-, 3-, and 4-jet production with a parton shower. 

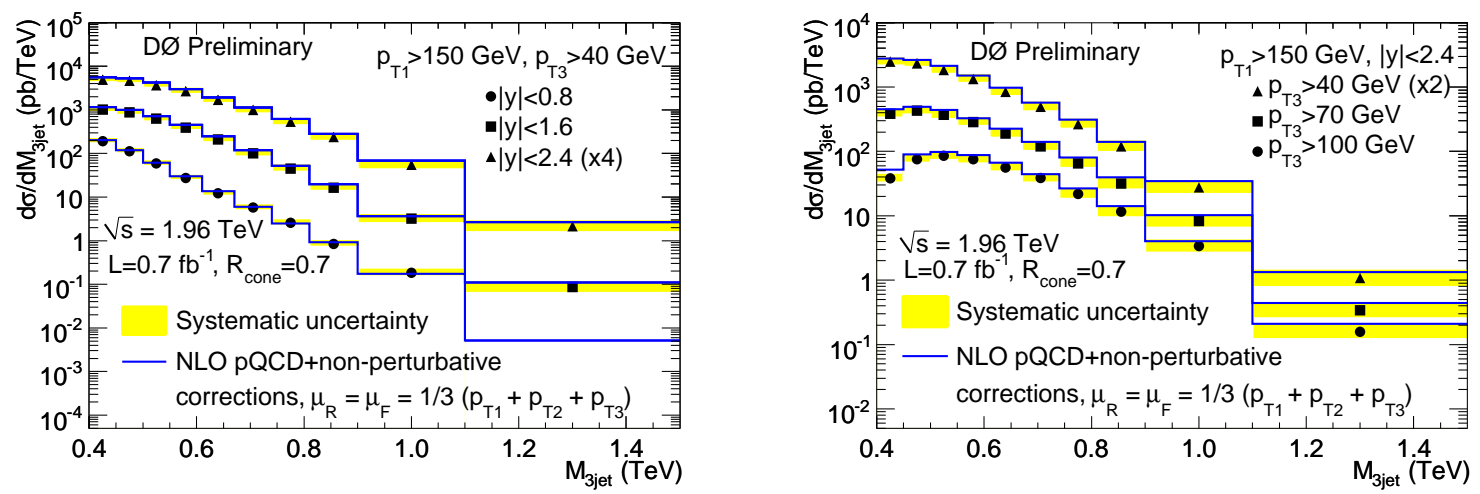

Figure 1: a) Three-jet mass cross section in regions of jet rapidities. The $|y|<2.4$ region is scaled by a factor of 4 for readability. Systematic uncertainty is shown by shaded band. Full lines correspond to the NLO calculations with NLOJET++ and MSTW2008 PDFs. No events are found in the highest $M_{3 \text { jet }}$ bin in $|y|<0.8$ region. b) Three-jet mass cross section in regions of the third jet transverse momenta. The $p_{T 3}>40 \mathrm{GeV}$ region is scaled by a factor of 2 for readability. Systematic uncertainty in all three-jet mass bins is shown by shaded band. Full lines correspond to the NLO calculations with NLOJET++ and MSTW2008 PDFs. In both figures, the dataset corresponds to an integrated luminosity of $0.7 \mathrm{fb}^{-1}$.

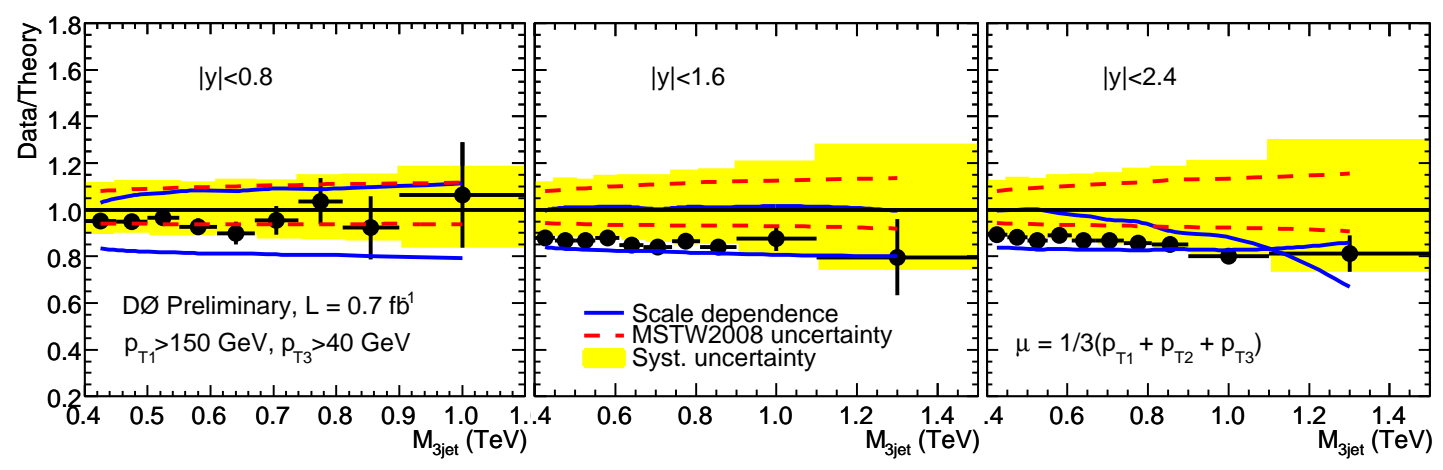

Figure 2: Data to theory ratio of the three-jet mass cross section in three regions of jet rapidities. The total systematic uncertainty is shown by a shaded band. The PDF uncertainty comes from the 20 MSTW2008NLO eigenvectors. The scale uncertainty is determined by varying the scale up and down by a factor of 2 .

In addition, the data are compared to predictions from the PYTHIA event generator (version 6.422). The matrix elements implemented in PYTHIA are only those for 2-jet production. All additional jet emissions are produced by a parton shower. There are two different implementations, a virtuality-ordered parton shower and a $p_{T}$-ordered one. Both are highly tunable and more than 50 tunes are provided in PYTHIA v6.422. All tunes studies here use the CTEQ5L PDFs [8].

In Fig. 3 the data are compared to PYTHIA tunes which use the $p_{T}$-ordered parton shower [9, 10]. These are tune "Professor pT0" [11] and two extreme tunes from the "Perugia" series of tunes [12], the tunes "Perugia hard" and "Perugia soft". All of these tunes give very different results for $R_{3 / 2}$ but all predict significantly higher ratios $R_{3 / 2}$ than what is seen in the data, even for the softest tune from the Perugia series. 


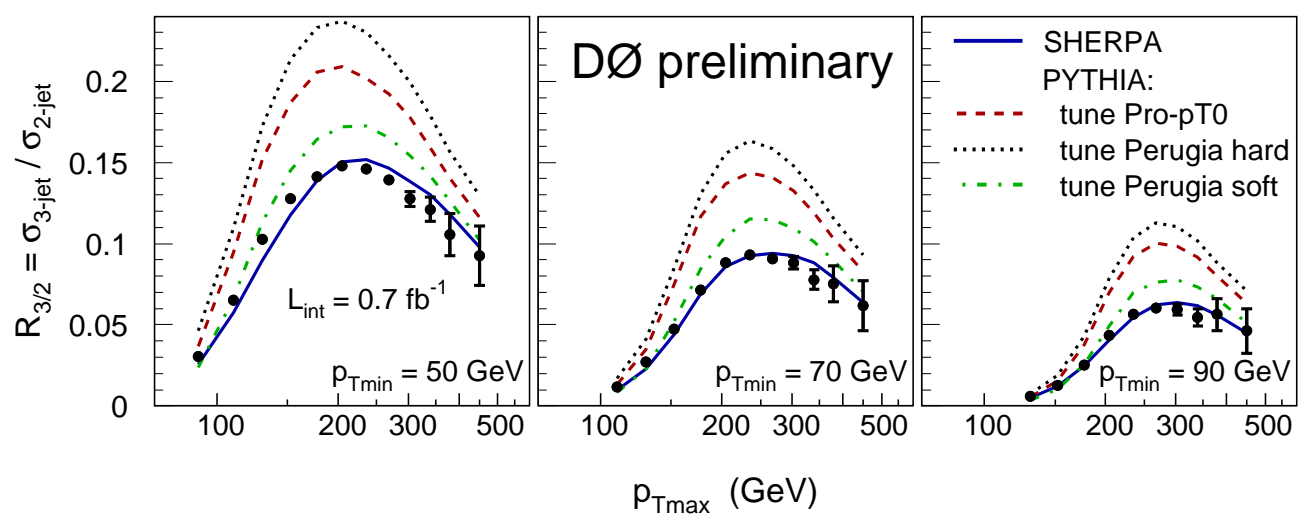

Figure 3: The ratio $R_{3 / 2}$ of trijet and dijet cross sections, measured as a function of the leading jet $p_{T}$ ( $\left.p_{T \max }\right)$ for different $p_{T \text { min }}$ requirements for the other jets. The inner error bars represent the statistical uncertainties while the total error bars represent the quadratic sums of statistical and systematic uncertainties. The predictions of SHERPA and PYTHIA (for three tunes using the $p_{T}$-ordered parton shower) are compared to the data.

\section{Angular Correlations in Minimum Bias Events}

While jet production is described reasonably well by perturbative QCD convoluted with PDFs, a variety of topics (usually grouped under the heading of "soft QCD processes") are not amenable to a pertubrative QCD calculation. These would include the development of showers in jets, the underlying events produced from the remnant partons of a collision and possibly initial state radiation, minimum bias events, etc. Models of these processes require comparison to experimental results in order to tune the model parameters. We have studied the angular separation in the detector transverse plane, $\Delta \phi$, between the leading track in the events ( $p_{T \text { lead }}$ and all other tracks in the event, as a way to characterize minimum bias events. This variable is then compared to several Monte Carlo tunes and models.

In order to create a sample of minimum bias events, we first select with exactly two muons with $p_{T}>2 \mathrm{GeV}$, both associated with the primary vertex of the event. We require that the event contains one more additional minimum bias vertices, with at least five tracks associated to each vertex. These minimum bias vertices are required to separated from the primary vertex by at $5 \mathrm{~cm}$, and to be within $20 \mathrm{~cm}$ of the center of the detector. All tracks are required to have $p_{T}>0.5 \mathrm{GeV}$ and to be within $|\eta|<2.0$. Approximately 4.3 million minimum bias vertices were found to satisfy these selection requirements.

For each minimum bias vertex, the angular separation between the track with the largest $p_{T}$ and every other track associated with the same minimum bias vertex is computed. The backgroundsubtracted distribution (using a polynomial fit to the distribution) is then normalized to the number of tracks, as only the shape of the resulting distribution is important for comparison to models. The normalized, background-subtracted $\Delta \phi$ distribution is shown in Fig. 4 , in bins of $\pi / 50$, for two different ranges of leading track $\eta$.

We have compared the distribution obtained from data with some tunes and models implemented in PYTHIA version 6.421 in two $|\eta|$ ranges: $|\eta|<1$, historically the region for which 

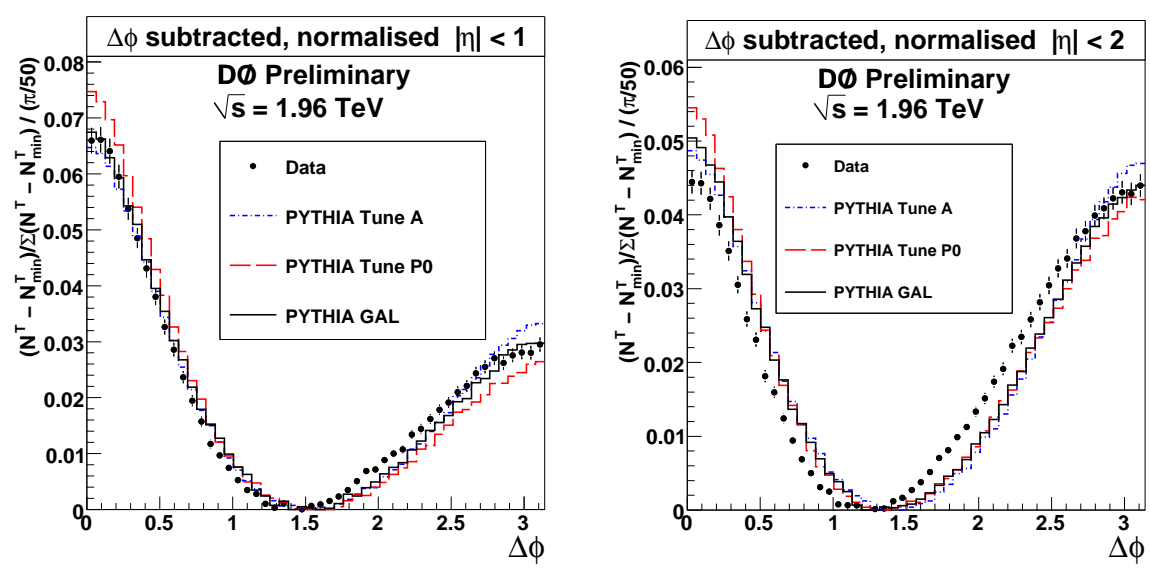

Figure 4: The normalized distribution of $\Delta \phi$ to the leading track in the same minimum bias vertex, for (a) $|\eta|<1.0$ and (b) $|\eta|<2.0$. Error bars include systematic uncertainties.

tuning data is provided [13], and $|\eta|<2$, the region accessible with the D0 detector. The comparison of the data $\Delta \phi$ distribution, subtracted and normalized, to three PYTHIA tune and model implementations (Tune A [14], the Perugia 0 tune (P0) [15], and the Generalized Area Law model of color reconnections (GAL) [16]) in those two $\eta \mid$ ranges are shown in Fig. 4 (a) and (b). Tune A uses $Q^{2}$-ordered parton showers, while tune $\mathrm{P} 0$ is a more recent tune which is an update of the First Sandhoff-Skands tune (S0) [17] and uses $p_{T}$-ordered showers. They both use the color-annealing model for color reconnections. GAL is based on tune S0 but uses the Generalized Area Law color reconnection model. In comparing the distributions in Fig. 4 (a) and (b), it is clear that the extended reach of the D0 detector allows us to access a region that affects the shape significantly and where the Monte Carlo tunes and models present large differences, increasing the tuning power.

\section{Summary}

In summary, we have presented the first measurement of the three-jet mass cross-section, and the ratio $R_{3 / 2}$ of trijet and dijet cross section in hadron-hadron collisions at a center of mass energy of $\sqrt{s}=1.96 \mathrm{TeV}$. The three-jet mass cross section is presented as a function of the leading jet $p_{T}$, for three regions of leading jet rapidity and three regions of the $p_{T}$ of the third jet. The ratio $R_{3 / 2}$ is presented for various $p_{T \min }$ requirements on the jets, as a function of the leading jet $p_{T}$.

In addition to our multijet results, we also have presented a new variable for investigating minimum bias and underlying events, based on the angular correlation between tracks. This new observable allows discriminative power between Monte Carlo models and tunes. While some some of the models studied describe the angular correlations better than other, none described the distributions well.

\section{References}

[1] V. M. Abazov et al. (D0 Collaboration), Nucl. Instrum. Methods Phys. Res. A 565, 463 (2006).

[2] G. C. Blazey et al., in Proceedings of the Workshop: QCD and Weak Boson Physics in Run II, edited by U. Baur, R.K. Ellis, and D. Zeppenfeld, (Batavia, Illinois, 2000) p. 47, see Section 3.5. 
[3] Z. Nagy, Phys. Rev. D 68, 094002 (2003); Z. Nagy, Phys. Rev. Lett. 88, 122003 (2002).

[4] A. D. Martin, W. J. Stirling, R. S. Thorne and G. Watt, Eur. Phys. J. C 63, 189 (2009).

[5] T. Sjöstrand et al., Comput. Phys. Commun. 135, 238 (2001).

[6] M. G. Albrow et al. (TeV4LHC QCD Working Group), arXiv:hep-ph/0610012.

[7] T. Gleisberg, S. Hoche, F. Krauss, M. Schonherr, S. Schumann, F. Siegert and J. Winter, JHEP 0902, 007 (2009).

[8] H. L. Lai et al. (CTEQ Collaboration), Eur. Phys. J. C 12, 375 (2000).

[9] T. Sjostrand and P. Z. Skands, Eur. Phys. J. C 39, 129 (2005).

[10] C. Buttar et al., arXiv:hep-ph/0604120.

[11] A. Buckley, H. Hoeth, H. Lacker, H. Schulz and E. von Seggern, arXiv:0906.0075[hep-ph].

[12] P. Z. Skands, arXiv:0905.3418[hep-ph].

[13] F. Abe, et al. (CDF Collaboration), Phys. Rev. Lett. 61, 1819 (1988). F. Abe, et al. (CDF Collaboration), Phys. Rev. D 41, 2330 (1990).

[14] R. D. Field (CDF Collaboration), in the Proceedings of APS / DPF / DPB Summer Study on the Future of Particle Physics (Snowmass 2001), Snowmass, Colorado, 30 Jun - 21 Jul 2001, pp P501

[15] P. Z. Skands, “The Perugia Tunes,” (arXiv:hep-ph/0905.3418).

[16] J. Rathsman, Phys. Lett. B 452 (1999) 364

[17] T. Sjostrand and P. Z. Skands, Eur. Phys. J. C 39 (2005) 129 